\title{
A model to predict the cell density and cell size distribution in nano-cellular foams
}

\author{
Irfan Khan ${ }^{\mathrm{a}, *}$, David Adrian ${ }^{\mathrm{a}}$, Stéphane Costeux ${ }^{\mathrm{b}}$ \\ ${ }^{a}$ Engineering 83 Process Sciences, The Dow Chemical Company, Midland, MI (48674) \\ ${ }^{b}$ Dow Building 83 Construction, The Dow Chemical Company, Midland, MI (48674)
}

\begin{abstract}
A numerical model is developed to simulate the simultaneous bubble nucleation and growth during depressurization of thermoplastic polymers saturated with supercritical blowing agents. Of particular importance is the ability of the model to predict the formation of nano-cellular foams, including the cell size distribution within the foam, based on the specific process conditions and polymer properties. Additionally the model differentiates between the "Free" and "Limited" expansion phases in the growth of a single bubble. Classical nucleation theory is used to predict nucleation rate and the popular "Influence Volume Approach" is used to determine the end of nucleation phase. By solving the mass, momentum and species conservation equations for each bubble, the model is capable of predicting bubble size distribution and bulk porosity.

It is found that by accurately capturing the concentration gradient of the blowing agent in the boundary layer surrounding the bubble and applying appropriate boundary conditions at different stages of bubble growth, the
\end{abstract}

\footnotetext{
*Corresponding Author. Tel.:+1 979-238-3538, Fax.:979-238-9674

Email address: ikhan@dow.com (Irfan Khan)
} 
model is able to accurately predict the conditions for making nano-cellular foams. Unlike micro-cellular foams the diffusion controlled period is short and the viscosity-controlled period is crucial to generating maximum nuclei density. Experimental data obtained by foaming acrylate copolymers with $\mathrm{CO}_{2}$ as the blowing agent compare well with model predictions of average cell size and porosity as well as cell size distribution. Furthermore, the effect of depressurization curve on the average cell size and cell size distribution are delineated.

Keywords: Classical Nucleation Theory, Simultaneous Nucleation and Bubble Growth, Finite-Element Method, Experimental Validation, Bubble Size Distribution.

\section{Introduction}

Exploiting the unique properties expected of nano-cellular foams can help solve challenging limitations in a number of fields including insulation and acoustics and is envisioned to be the new frontier in foam science (Costeux, 2014). For instance, polymeric foams with small cell size $(<200 \mathrm{~nm})$ and high void fraction $(>95 \%)$ are expected to provide significant improvement in insulation efficiency (Notario et al., 2014; Forest et al., 2014), beyond current industry standards. However, the process of polymeric foaming is characterized by complex interdependence of the polymer+blowing agent thermodynamic properties in addition to rapid material transport. Understanding the mechanisms that govern thermodynamic and transport phenomena is critical to achieving better control of cell size and cell number density in thermoplastic foams. 
Experimental work has been invaluable in providing limitations and guidelines for the choice of processing conditions and polymer properties in making micro-cellular and nano-cellular foams (Han and Han, 1990; Park et al., 1995; Taki et al., 2003; Costeux, 2014). However due to the small time scales and rapid mass transport involved, they cannot probe the necessary resolution for a fundamental understanding of the process. Mathematical modeling tools have the ability to investigate the phenomena at spatial and temporal resolutions inaccessible to experiments. There has been extensive work in this area starting with Street, who investigated the single bubble growth, theoretically for both Newtonian (Street, 1968) and non-Newtonian fluids (Street et al., 1971). Amon and Denson (1984)'s "Cell model" was helpful in understanding the effect of limited gas supply on bubble growth. Work done by Arefmanesh et al. (1992); Venerus et al. (1998) provided further insight into the dynamics of single bubble growth including the understanding of lower and upper bounds of bubble growth rates (defined as the rate of increase in bubble diameter).

The "Influence Volume Approach" (IVA) proposed by Shafi et al. (1996, 1997) was the first time a model could simulate nucleation and bubble growth simultaneously. Although the model made many assumptions such as instantaneous depressurization, same initial conditions for all nucleated bubbles and the non-existence of a limited growth period, the model could connect the final foam properties (nucleation density, porosity and bubble size distribution $(\mathrm{BSD}))$ to foaming conditions (soak temperature, soak pressure, depressurization rate) and material properties (viscosity, surface tension, solubility of gas etc.). 
In subsequent work, other models have been proposed to connect process conditions and material properties to the foam properties, either by modifying the IVA or proposing similar models. Assuming instantaneous depressurization, Feng and Bartelo (2004) simulated nucleation and bubble growth of micro-cellular foams in viscoelastic fluids using a simpler nucleation model. Leung et al. (2006a) coupled a nucleation model using a modified classical nucleation theory (CNT) with a bubble growth model to simulate simultaneous nucleation and bubble growth. Unlike most of the previous work they used a finite depressurization rate and thus were able to capture the slow increase in the nucleation rate and then its later decrease, which leads to a normal BSD. Taki (2008) used a numerical model based on the IVA and CNT to investigate the effect of pressure release rate on cell growth rate. He observed the presence of three distinct regimes during cell growth; the viscosity-limited initial regime, the transitional regime and finally the diffusion-limited regime. Their work was focused on micro-cellular foams and assumed that the concentration of blowing agent in the influence volume is given by a chosen polynomial function.

All the above mentioned models do not differentiate between two stages, namely when the Influence Volume (IV) surrounding each bubble expands freely (Free Expansion) and when it starts to overlap with other bubbles (Limited Expansion) leading to rapid decrease in the concentration gradient. Some of the models mentioned above assume that the concentration profile of blowing agent in the influence volume is represented by a polynomial. This assumption is suitable for foaming of micro-cellular foams where the diffusioncontrolled bubble growth period dominates. In nano-cellular foaming such an 
approximation grossly under-estimates the mass transfer during the critical viscosity-controlled period. A systematic method of calculating the BSD from a numerical simulation that has been validated with experimentally obtained BSD is thus lacking.

In this work, the IVA is used along with CNT to model nucleation and bubble growth simultaneously. Furthermore the Free Expansion and Limited Expansion periods of the foaming process are differentiated with appropriate boundary conditions for bubble growth. By using a finite depressurization rate and considering independent growth rate for all bubbles, the model is able to predict the bubble size distribution. Experimental data gathered on nano-cellular foams made from acrylate copolymers with $\mathrm{CO}_{2}$ as the blowing agent are used for comparison with model predictions. Furthermore, the effect of depressurization profile on BSD is investigated and the results are described.

\section{Methodology}

The work presented here is based on the principles of the "Influence Volume Approach" (IVA), that was first proposed by Shafi et al. (1996),Shafi et al. (1997). The IVA presents a logical means of predicting the end of nucleation during the foaming process and thus allows for modeling the nucleation and bubble growth phases simultaneously as it occurs during any foaming process. According to the IVA, surrounding each bubble is a region with de-

pleted blowing agent concentration. This region, which is called the Influence Volume (IV) grows as more dissolved gas diffuses into the bubble during the bubble growth phase. Furthermore, because of the lower gas concentration in 
this region, the probability of subsequent nucleation in this region decreases. Because of these assumptions, the model distinguishes the nucleation time which is governed by the availability of supersaturated sites in the polymer from the depressurization time, unlike most of the other models (Leung et al., 2007; Taki, 2008; Feng and Bartelo, 2004).

The model assumes that at time $t=0$, the polymer is saturated with gas at concentration $C_{o}$ at some elevated pressure $P_{o}$. When the pressure is released the gas supersaturates and evolves out of the polymer causing nucleation of bubbles and their subsequent growth. This period of bubble growth is characterized by the bubble growth rate increasing with increasing influence volume (IV). This is called the "Free Expansion Phase". Nucleation continues till the combined IV of all the bubbles spreads across the entire polymer melt volume and ends when there is no Uninfluenced Volume (UIV) left in the polymer melt.

Following the end of nucleation, the available dissolved gas for each bubble is fixed as the IV stops growing. This period is characterized by a decreasing bubble growth rate and is called the "Limited Expansion Phase" (Mao et al., 2006). Bubble growth continues till either the pressure inside the bubble reaches ambient pressure or the glass transition temperature $\left(T_{g}\right)$ of the polymer-dissolved gas mixture increases above the local temperature.

Additionally the following simplifying assumptions are also made

1. The bubble is spherically symmetric after nucleation and remains so during the entire growth. Interactions between bubbles are assumed to occur only through the influence volume. Bubbles cannot coalesce.

2. The gas pressure in the bubble $\left(P_{b u b}\right)$ is related to the gas concentration 
$(C(R, t))$ at the bubble surface by Equation 1 , where $K_{H}$ is the Henry's constant.

$$
C(R, t)=K_{H} P_{b u b}
$$

3. The process is assumed to be isothermal

4. The polymer $+\mathrm{CO}_{2}$ is a viscous fluid, its viscosity is shear rate dependent in accordance with the Cross model

\subsection{Governing equations for bubble growth}

As mentioned earlier, we make the assumption that bubble growth is spherically symmetric, which along with the assumption of an incompressible polymer fluid leads to the equation

$$
v_{r}(r)=\frac{\dot{R} R^{2}}{r^{2}}
$$

where $v_{r}$ is the radial component of polymer velocity surrounding the bubble, $R$ is the bubble radius and $r$ is the distance from bubble center as shown in Figure 1. $\dot{R}$ represents the time derivative of $R$.

A quasi-static momentum balance across the melt phase combined with the jump linear momentum across the bubble-liquid interface gives

$$
P_{b u b}-P_{l i q}-\frac{2 \sigma}{R}=\int_{R}^{\infty} \frac{\tau_{r r}-\tau_{\theta \theta}}{r} d r
$$

where $\sigma$ is the interfacial tension between the gas and liquid phases and $\tau_{r r}$ and $\tau_{\theta \theta}$ are the radial and tangential components of the stress tensor, respectively. The stress tensors $\tau_{r r}$ and $\tau_{\theta \theta}$ are given as

$$
\tau_{r r}=\mu\left[2 \frac{\partial v_{r}}{\partial r}-\frac{2}{3} \nabla \cdot \mathbf{V}\right]
$$




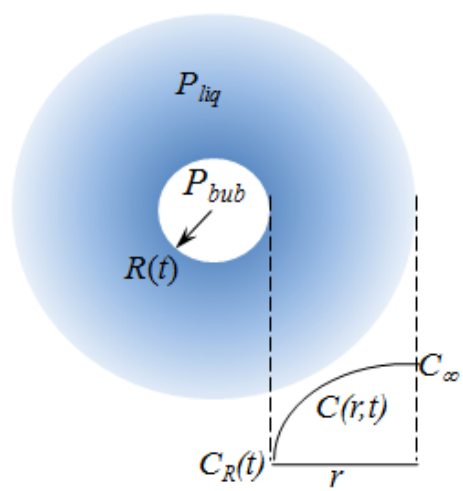

Figure 1: The influence volume surrounding the bubble has a depleted concentration of the blowing agent.

$$
\tau_{\theta \theta}=\mu\left[2\left(\frac{1}{r} \frac{\partial v_{\theta}}{\partial \theta}+\frac{v_{r}}{r}\right)-\frac{2}{3} \nabla \cdot \mathbf{V}\right]
$$

Typically, the dissolution of blowing agent depresses the glass transition temperature of the polymer causing it to be in a plastic state and significantly changing the viscosity of the system. In order to take this into account, in this work, viscosity $(\mu)$ is considered to be a function of temperature, pressure and strain rate and is calculated at each time step. The Cross function is employed to predict viscosity with respect to strain rate and is given as

$$
\eta(\dot{\gamma})=\frac{\eta_{o}}{1+(\lambda \dot{\gamma})^{n}}
$$

where $\eta_{o}$ is the zero shear viscosity, $\lambda$ and $n$ are the cross model parameters. Note that $\eta_{o}$ and $\lambda$ are both function of temperature and $\mathrm{CO}_{2}$ mass fraction.

Thus equations 4,5 were evaluated assuming a known viscosity and substituted into Equation 3 to give the governing equation for the growth rate of the bubble

$$
\frac{d R}{d t}=\frac{\left(P_{b u b}-P_{l i q}\right) R}{4 \mu}-\frac{\sigma}{2 \mu}
$$


Although the above equation is derived assuming a Newtonian fluid, the viscosity in Equation 7 is evaluated at every timestep based on the strain rate and blowing agent concentration at the bubble interface. A simplification done to take into account the $T_{g}$ depression due to the blowing agent and the shear thinning effect.

It is assumed that at time $t=0$, the initial bubble radius is given by the critical bubble radius $R_{c r}$ as,

$$
R(t=0)=R_{c r}=\frac{2 \sigma}{\left(P_{b u b}-P_{l i q}\right)}
$$

The assumption of a spherically symmetric bubble reduces the governing equation for the concentration of gas in the melt surrounding the bubble $(C(r, t))$ to a one-dimensional equation and is given as

$$
\frac{\partial C}{d t}+v_{r} \frac{\partial C}{d r}=\frac{\mathcal{D}}{r^{2}} \frac{\partial}{\partial r}\left(r^{2} \frac{\partial C}{\partial r}\right)
$$

where $\mathcal{D}$ is the diffusivity of the gas in the polymer, which is a function of temperature and pressure. The initial condition for the above equation is given by $C(r, 0)=C_{o}$. The boundary conditions for Equation 9 is given by a specified concentration at the bubble surface given as $C(R, t)=K_{H} P_{b u b}$. The other boundary condition at $R=\infty$ depends on whether it is a Free expansion phase or the Limited expansion phase.

Finally the mass balance on the bubble surface results in a relationship between the bubble pressure $P_{b u b}$ and the bubble radius $R$ and is given as

$$
\frac{d}{d t}\left(\frac{4 \pi R^{3}}{3} \frac{P_{b u b}}{\mathcal{R} T}\right)=\mathcal{D} 4 \pi R^{2} \frac{d C}{d r}
$$

where $\mathcal{R}$ is the universal gas constant and $T$ is the gas temperature in Kelvin. The simultaneous solution of equations 7, 9 and 10 results in the determina- 
tion of the bubble pressure $\left(P_{b u b}\right)$, bubble radius $\left(R_{b u b}\right)$ and the gas concentration $(C(r, t))$.

\subsection{Nucleation}

There has been a lot of debate on the appropriate nucleation model to use for a foaming process. Classical Nucleation Theory (CNT) formed the basis of the nucleation model in the initial investigations (Shafi et al., 1996, 1997). Modifications were made to the CNT to take into account effect of stresses (Lee, 1995) and varying contact angle (Leung et al., 2006a). Recent work based on Density Functional Theory (DFT) combined with minimum free energy path calculations using the String Method (Xu et al., 2013b,a) has shown that CNT severely underpredicts the available free energy for a supercritical blowing agent dissolved in a polymer matrix. The error in assuming a flat surface interfacial tension on the predictions of CNT were delineated by Kim et al. (2011).

Considering that CNT underpredicts the nucleation rate, in this work the CNT is used with a fitting parameter, an approach previously employed by Taki (2008). Due to the high depressurization rate used in this work, it is assumed that homogeneous nucleation is the primary mode of creating new nuclei. Thus following Blander and Katz (1975), the rate of nucleation $\left(\frac{d N}{d t}\right)$, is given as

$$
\frac{d N}{d t}=A_{1} \mathcal{N}\left[\frac{2 \sigma}{\pi m}\right]^{0.5} \exp \left(-\frac{16 A_{2} \pi \sigma^{3}}{3 k T\left[P_{b u b}-P_{l i q}\right]^{2}}\right),
$$

where $\mathcal{N}$ is the number of dissolved gas molecules per unit volume of the primary phase, $m$ is the mass of the gas molecule, $\sigma$ is the interfacial tension between the blowing agent and the saturated polymer, $k$ is the Boltzmann 
constant and and the parameters $A_{1}, A_{2}$ are obtained by fitting the model to experiments. These fitting parameters are considered to be constant for a given polymer+blowing agent system. A similar approach was used by Costeux et al. (2014) to compare foaming model predictions with experimental data. They found that the CNT yielded foaming predictions that matched resonably well with the experimental data.

As per the IVA, new nuclei are only generated in the uninfluenced volume (UIV). Thus the total number of nuclei $\left(N_{\text {tot }}(t)\right)$ generated at time $t$ are given by

$$
N_{t o t}(t)=\int_{0}^{t} \frac{d N(\tau)}{d t} U I V(\tau) d \tau
$$

where $\frac{d N(t)}{d t}$ represents the nucleation rate at time $t$ and $U I V$ is the remaining uninfluenced volume given by

$$
U I V(t)=V_{o}-\int_{0}^{t} \frac{d N}{d t}(t-\tau) U I V(t-\tau) V_{c b}(\tau) d \tau,
$$

where $V_{o}$ is the total melt volume at time $t=0$ and $V_{c b}$ is the influence volume surrounding a bubble.

\subsection{Evaluating the Influence Volume}

Accurate evaluation of the influence volume and the concentration profile $(C(r, t))$ is critical for determining the nucleation end time and also to calculate the correct bubble growth rate. In the literature, the concentration profile has been evaluated using either finite element or finite volume methods (Arefmanesh et al., 1992; Feng and Bartelo, 2004; Leung et al., 2006b) or using a polynomial function that satisfies the imposed boundary conditions. Han and Yoo (1981) used a quadratic polynomial to approximate the concentration profile. Payvar (1987) used a slightly modified form of the Han 
and Yoo (1981)'s quadratic polynomial. Shafi et al. (1996) used a $12^{\text {th }}$ order polynomial in $r$ and claimed that the results did not differ when compared to the solution of a finite element method. Shimoda et al. (2001) followed Shafi et al. (1996) and used the same polynomial function to predict the cell size in an extrusion process.

Taki (2008) compared the models by Han and Yoo (1981),Payvar (1987) and Shafi et al. (1996) for single bubble growth rate to experimental data for a Polypropylene $+\mathrm{CO}_{2}$ system. Although they found that the model by Han and Yoo (1981) best matched experimental data, they also noted that none of the models could fit different polymer+blowing agent systems.

The challenge in using a single approximate function for the concentration profile $\left(C(r, t)\right.$ is the large change in the Peclet number $\left(P e=\frac{\dot{R} R}{\mathcal{D}}\right)$ that occurs during the growth of the bubbles. Following bubble nucleation $(R \approx$ $\left.10^{-9} ; \dot{R} \approx 10^{-5} ; \mathcal{D} \approx 10^{-10}\right) P e \ll 1$, after sufficient bubble growth $(R \approx$ $10^{-7} ; \dot{R} \approx 10^{-3} ; \mathcal{D} \approx 10^{-10}$ ) Pe $\approx 1$. Bubble growth becomes diffusion limited further along and the $P e<1$.

To avoid the uncertainty accompanying the choice of approximating function for the concentration profile, in this work the Galerkin finite element method (FEM) is used to solve Equation 9.

Since the domain of interest $(R(t)<r<\infty, t)$ changes with time, Equation 9 was transformed to a stationary coordinates $(1<y<\infty, t)$ using the transformation $y=\frac{r}{R}$.

The transformed species transport equation is given by

$$
\frac{\partial C}{\partial t}=\frac{2 \mathcal{D}}{R^{2}} \frac{1}{y} \frac{\partial C}{\partial y}+\frac{\dot{R}}{R}\left[y-\frac{1}{y^{2}}\right] \frac{\partial C}{\partial y}+\frac{\mathcal{D}}{R^{2}} \frac{\partial C}{\partial y}
$$

The Galerkin method is used to discretize Equation 14. Quadratic ele- 
ments are used to approximate the concentration and are given by

$$
C_{e}(y, t)=\sum_{i=1}^{\mathfrak{N}_{p e}} C_{i}^{t} \phi_{i}(y)
$$

where $\phi_{i}(y)$ is the quadratic Lagrange polynomial function, $C_{i}^{t}$ is the value of concentration at node $i$ at time $t$ and $\mathfrak{N}_{p e}$ is the number of nodes per element.

Substituting the approximation into the Galerkin weighted integral form of Equation 14 and assembling the resulting equation for all the nodes, one obtains the system of equation given by

$$
\mathbf{M} \frac{d \bar{C}}{d t}+\left\{\mathbf{K}^{1}+\mathbf{K}^{2}+\mathbf{K}^{3}\right\} \bar{C}=0
$$

where $\mathbf{M}$ is the mass matrix, $\mathbf{K}=\left\{\mathbf{K}^{\mathbf{1}}+\mathbf{K}^{\mathbf{2}}+\mathbf{K}^{\mathbf{3}}\right\}$ is the stiffness matrix and $\bar{C}$ is the concentration vector. The matrices are evaluated as

$$
\begin{aligned}
M_{i j} & =\int_{y_{e}} \phi_{i} \phi_{j} d y, \\
K_{i j}^{1} & =\frac{2 \mathcal{D}}{R} \int_{y_{e}} \frac{\phi_{i}}{y} \frac{\partial \phi_{j}}{\partial y} d y, \\
K_{i j}^{2} & =\frac{\dot{R}}{R} \int_{y_{e}}\left(y-\frac{1}{y}\right) \phi_{i} \frac{\partial \phi_{j}}{\partial y} d y, \\
K_{i j}^{3} & =\frac{\mathcal{D}}{R^{2}} \int_{y_{e}} \frac{\partial \phi_{i}}{\partial y} \frac{\partial \phi_{j}}{\partial y} d y,
\end{aligned}
$$

where $y_{e}$ is the element over which the integration is performed. In the above equation, $i$ and $j$ represent the local node number of the element $e$ which are connected to the global node numbering system as

$$
i_{g}=(e-1) *\left(\mathfrak{N}_{p e}-1\right)+i_{l}
$$

where $i_{g}$ is the global node number, $e$ is the element number, $\mathfrak{N}_{p e}$ represents nodes-per-element and $i_{l}$ is the local node number. The integrals in Equation 
17 are evaluated using 3-point Gaussian quadrature. Iso-parametric mapping allows to transform the element in real space onto a master element with coordinates of $(-1,0,1)$.

The global mass and stiffness matrices are constructed by calculating the element-wise contributions and sewing together following the above local to global node numbering convention.

A semi-implicit time stepping algorithm is used to integrate Equation 16 resulting in

$$
\left[\mathbf{M}+a \Delta t \mathbf{K}_{t+1}\right] \vec{c}_{t+1}=\left[\mathbf{M}-(1-a) \Delta t \mathbf{K}_{t}\right] \vec{c}_{t}
$$

where $\Delta t$ is the time-step and $a$ was chosen to be 0.5 (Crank-Nicolson scheme).

\subsection{Free Expansion Phase}

During the nucleation period, the average bubble diameter is small (5$10 \mathrm{~nm}$ ) leading to a large gap between bubbles $(\approx 100 \mathrm{~nm})$. Thus it is assumed that the bubbles do not interact in any form. Therefore, the influence volume of all the bubbles expand freely. The appropriate boundary conditions in this period for Equation 9 is given by

$$
\begin{gathered}
C(R, t)=k_{H} P_{b u b} \\
C(\infty, t)=C_{o}
\end{gathered}
$$

The influence volume $\left(V_{c b}\right)$ is calculated as

$$
V_{c b}=\frac{4 \pi}{3}\left(R_{\infty}^{3}-R^{3}\right)
$$

where $R_{\infty}$ is chosen such that $C\left(R_{\infty}, t\right)=0.98 C_{o}$. 


\subsection{Limited Expansion Phase}

At the end of nucleation, the combined influence volume of all the bubbles $I V=\sum_{i=1}^{t o t b u b} V_{I V}^{i}$ is equal to the volume of polymer and remaining dissolved blowing agent. At this point the influence volume of the bubbles starts to overlap with each other. Thus at the end of the influence volume $\left(R_{\infty}\right)$ the concentration $\left(C\left(R_{\infty}, t\right)\right)$ is lower than $\kappa C_{o}$. This boundary condition is implemented by assuming that $R_{\infty}$ varies with time as

$$
R_{\infty}^{t+\Delta t}=R_{\infty}^{t}+\Delta t \frac{\dot{R} R^{2}}{\left(R_{\infty}^{t}\right)^{2}},
$$

which ensures the conservation of blowing agent in the remaining influence volume. Proof of mass conservation through this boundary condition is provided in supplemental material. Furthermore a zero gradient boundary conditions is imposed at $R_{\infty}$ given by

$$
\frac{\partial C}{\partial r}\left(R_{\infty}, t\right)=0
$$

\subsection{Algorithm}

Figure 2 shows the flow chart of the algorithm used in this work. The work of Leung et al. (2006a) found that assuming the saturation pressure to be constant $\left(P_{\text {sat }}=P_{o}\right)$ during nucleation, leads to significant over prediction of the nucleation rate. Thus in this work, the value of $P_{\text {sat }}$ is evaluated at every time-step based on the concentration of blowing agent.

Furthermore, in order to keep track of the cell size distribution, a fixed number $(Q)$ of bins are created, represented as $b_{i}$. The bin $b_{i}$ keeps track of all the bubbles generated within a time frame given by $\left(t_{i}, t_{i+1}\right)$, where

$0 \leq i \leq Q-1$. Each bin contains bubbles nucleated in that time frame and 


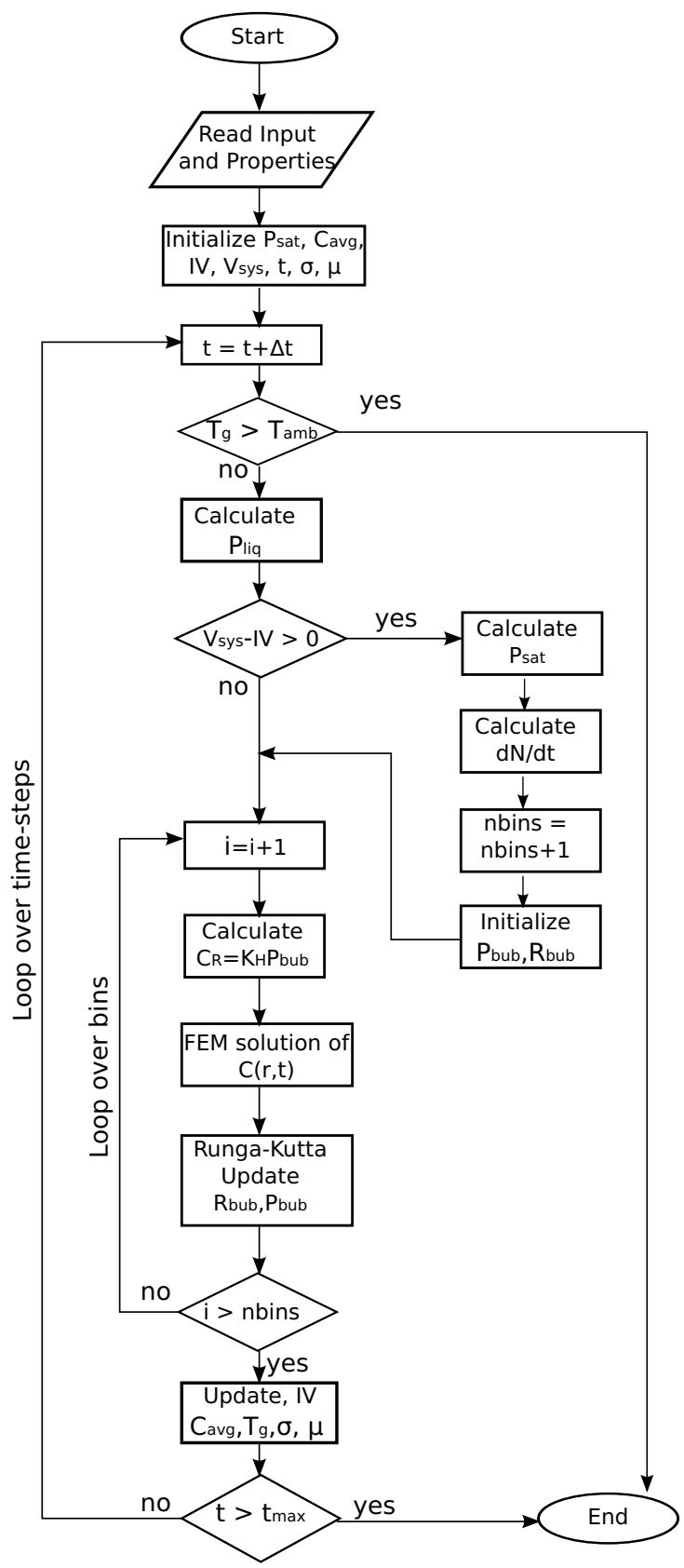

Figure 2: Flow Chart of the algorithm to simulate simultaneous nucleation and bubble growth in thermoplastic foams. 
assigns an average bubble radius $\left(R_{\text {avg }}^{i}\right)$ and bubble pressure $\left(P_{\text {avg }}^{i}\right)$ based on mass conservation. This is represented by the loop over the bins $(i)$ in Figure 2 .

Because of the rapid change in the Peclet number as mentioned earlier, a small time-step in needed to capture the concentration profile accurately and also to avoid introducing instability in the bubble growth. In this work, a timestep of $10^{-9}$ seconds is used. Additionally a $4^{\text {th }}$ order Runge-Kutta method is used to solve equations 10 and 7 in a coupled fashion. $Q$ is set to 1000 bins for the simulation results presented here.

\section{Physical Properties}

The work in this paper is an attempt to improve the foaming model presented by Costeux et al. (2014) by incorporating the Free and Limited expansion phases and improving the prediction of the BSD. The polymer/gas system considered for the modeling work is a copolymer of Methyl-methacrylate (MMA) with 9 wt\% Ethylacrylate (EA), referred to as MMA-EA9, with $\mathrm{CO}_{2}$ as the blowing agent system. Additional details about the polymer and its foaming behavior can be found elsewhere (Costeux et al., 2013).

The model requires data for $\mathrm{CO}_{2}$ solubility $\left(\mathrm{Y}_{\mathrm{CO}_{2}}\right)$ and diffusivity $(\mathcal{D})$ in the polymer, along with the glass transition temperature $\left(T_{g}\right)$, viscosity $(\mu(\dot{\gamma}))$ and interfacial tension $(\sigma)$ of the polymer $+\mathrm{CO}_{2}$ system as a function of temperature, pressure and $\mathrm{CO}_{2}$ mass fraction. Gathering such data for the polymer $+\mathrm{CO}_{2}$ system, especially at the $\mathrm{CO}_{2}$ critical temperature and pressure is very challenging and prone to errors due to buoyancy effects. Therefore some approximations were made in evaluating these properties. 
Table 1: Values of the physical properties and parameters used in foaming model at $35^{\circ} \mathrm{C} 30 M P a$.

\begin{tabular}{ll}
\hline Solubility, $\mathrm{Y}_{\mathrm{CO}_{2}}$ & $33.2 \mathrm{wt} \%$ \\
Diffusivity, $\mathcal{D}$ & $1.1 \times 10^{-10} \mathrm{~m}^{2} / \mathrm{s}$ \\
Henry's constant, $K_{H}$ & $0.028 \mathrm{~mol} / \mathrm{L} . \mathrm{atm}$ \\
Interfacial Tension, $\sigma$ & $4.9 \mathrm{mN} / \mathrm{m}$ \\
Zero-shear viscosity, $\lambda_{o}$ & $2730 \mathrm{kPa.s}$ \\
Glass transition, $T_{g}$ & $7^{\circ} \mathrm{C}$ \\
Cross parameter, $\lambda$ & $44.5 \mathrm{~s}$ \\
Cross parameter, $n$ & 0.656 \\
\hline
\end{tabular}

For instance the solubility and interfacial tension data was extrapolated using modeling tools that combined PC-SAFT equation of state with the density gradient theory (Xu et al., 2012). $T_{g}$ data was obtained from Condo and Johnston (1994). Diffusivity of $\mathrm{CO}_{2}$ is the polymer was obtained from Nilsson et al. (2013). Viscosity as a function of shear rate was obtained using a sliding plate rheometer and fit to a Cross model given in Equation 6. Shift factors for pressure, temperature and $\mathrm{CO}_{2}$ concentration were determined by the method proposed by Park and Dealy (2006).

Table 1 contains the values of the above mentioned physical properties and parameters for the MMA-EA9 copolymer saturated with $\mathrm{CO}_{2}$ at the temperature of $35^{\circ} \mathrm{C}$ and pressure $30 \mathrm{MPa}$.

\section{Single bubble growth}

To illustrate the Free and Limited expansion boundary conditions and the effect of using the FEM for resolving the concentration profile in the 
IV, the growth behavior of a single bubble is presented here. The results presented in the following track a single bubble nucleated at the start of the depressurization. The depressurization rate is approximated using an exponential function given as

$$
P_{\text {liq }}(t)=P_{\text {soak }} \exp \left(\frac{\log P_{\text {amb }}-\log P_{\text {soak }}}{t_{\text {dep }}} \cdot t\right)
$$

where $P_{a m b}$ is the ambient pressure and $t_{d e p}$ is the depressurization time.

Due to the rapid depressurization used in these simulations the bubble growth period is considerably shorter $(20 \mathrm{~ms})$ than those presented in literature (Shafi et al., 1996; Leung et al., 2006a; Taki, 2008). Time is nondimensionalized as

$$
t^{*}=\frac{t D}{\left(R_{c r}^{o}\right)^{2}},
$$

where $R_{c r}^{o}$ is an average critical radius of the bubble chosen to be $2 \mathrm{~nm}$. In Equation 8, $P_{l i q}$ is changing with time, thus $R_{c r}^{o}$ also changes with time. Bubble radius is non-dimensionalized as

$$
R^{*}=\frac{R}{R_{c r}^{o}} .
$$

Bubble pressure and concentration in the surrounding melt are non-dimensionalized as

$$
\begin{gathered}
P^{*}=\frac{P_{b u b}-P_{a m b}}{P_{\text {soak }}-P_{a m b}}, \\
C^{*}=\frac{C(r, t)-k_{H} P_{a m b}}{C_{o}-k_{H} P_{a m b}},
\end{gathered}
$$

respectively, where $P_{a m b}$ is the ambient pressure $(1.013 P a)$.

Figure 3a shows the concentration profile with respect to non-dimensional position $y=r / R_{b u b}$ at time $t^{*}=250$ obtained using FEM, Han \& Yoo 
function and Shafi et.al. function. Clearly there is considerable difference in the concentration profile as predicted by Shafi et.al. and Han \& Yoo models compared to the FEM predictions. Similarly there is considerable difference in the predictions of concentration gradients (Figure 3b) during the Free Expansion period; excluding the early part of bubble growth where diffusion is insignificant. It is seen that the polynomial functions underpredict the concentration gradient, resulting in considerable error in bubble growth and influence volume predictions.

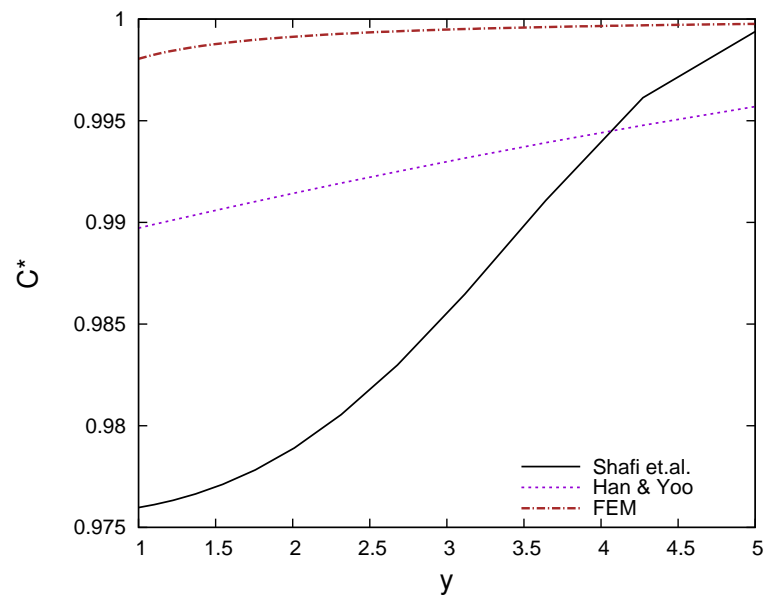

(a) Concentration profile

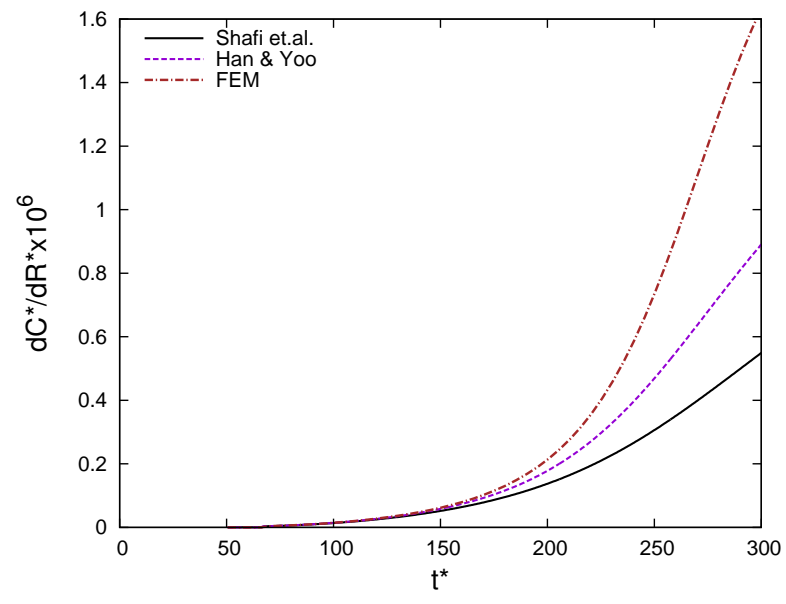

(b) Concentration gradient

Figure 3: Comparison of FEM with Shafi et.al. and Han \& Yoo polynomial functions to predict concentration profile and concentration gradient.

Figures 4 and 5 show the plots of bubble radius $(R)$, bubble pressure $\left(P_{b u b}\right)$, bubble growth rate $\left(\frac{d R}{d t}\right)$ and concentration gradient at the bubble surface $\left(\left.\frac{d C}{d r}\right|_{r=R}\right)$ with respect to time. The Free Expansion phase is represented by closed symbols and the Limited Expansion phase is represented by 
open symbols.

The general behavior of bubble growth as seen in Figure 4a is similar to that reported in literature. The growth of the new bubble starts relatively slow but gains momentum because of the increasing pressure difference $\left(P_{b u b}-P_{l i q}\right.$, Figure $\left.4 \mathrm{~b}\right)$. During this period mass diffusion is very slow mainly due to the very small value of bubble surface area. The pressure difference drives the increase in concentration gradient which increases by several orders of magnitude (Figure 5b). This rapid increase causes the bubble growth rate also to increase considerably (Figure 5a). Equation 7, which governs the growth rate of a bubble due to an imbalance in bubble internal and external pressure, shows that more than surface tension, the melt viscosity is the limiting criteria for bubble growth during this initial period.

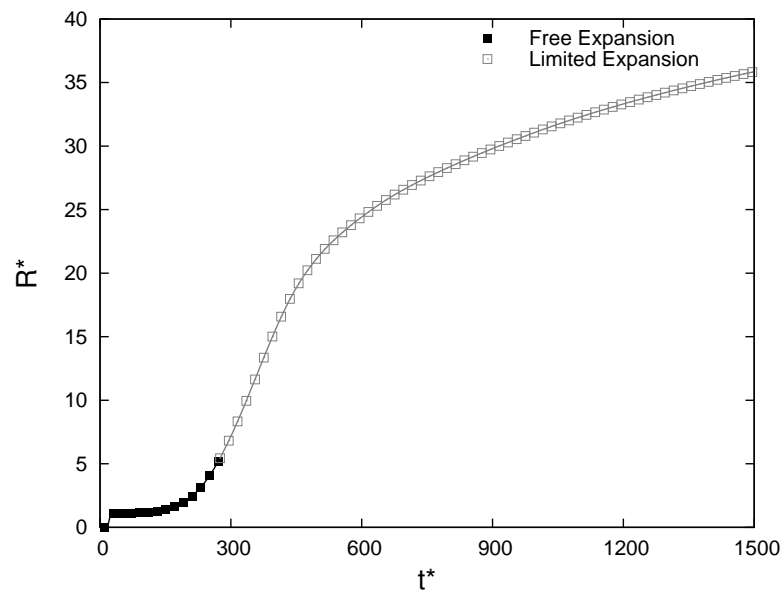

(a) Bubble radius $\left(R^{*}\right)$ w.r.t time

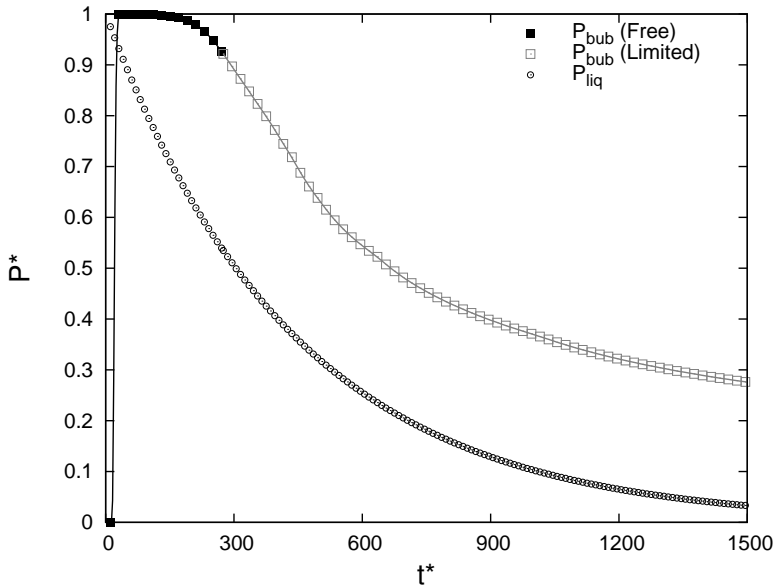

(b) Bubble pressure $\left(P^{*}\right)$ w.r.t time

Figure 4: Plots of bubble radius and pressure w.r.t time.

As new bubbles nucleate and grow, the Uninfluenced Volume decreases. 


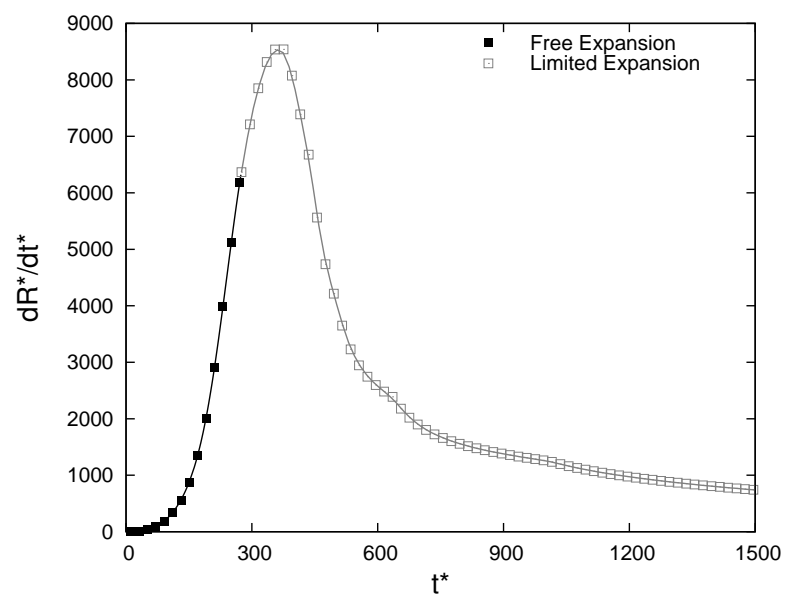

(a) Bubble growth rate $\left(\frac{d R^{*}}{d t}\right)$ w.r.t time

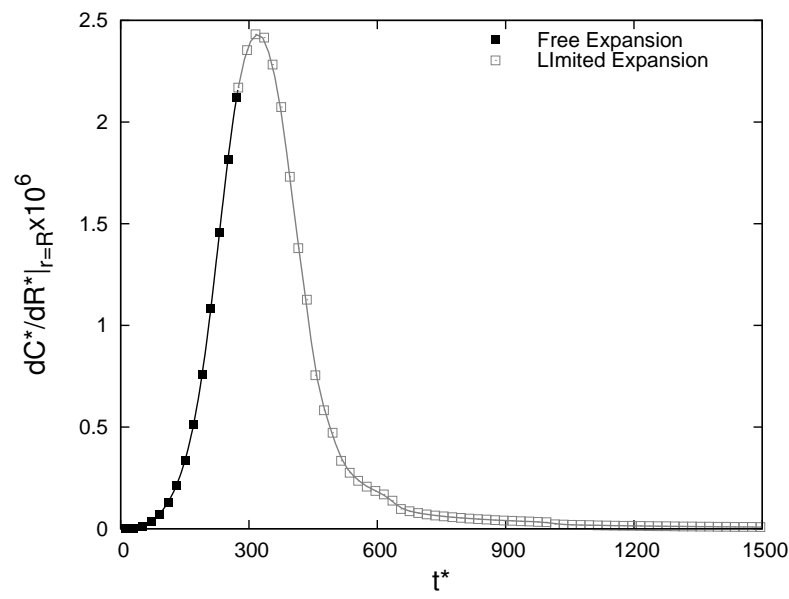

(b) Concentration gradient at bubble edge $\left(\left.\frac{d C^{*}}{d r}\right|_{r=R}\right)$ w.r.t time

Figure 5: Variation of the bubble parameters w.r.t time.

Eventually UIV $=0.0$ and the bubble enters the Limited Growth phase. As Figure 6a shows, the Limited Expansion boundary condition ensures that there is no increase in the available influence volume for a bubble after the end of the free expansion phase. Although in principle, there should be a decrease in the influence volume surrounding the bubble as the remaining blowing agent gets diffused into the bubble. However, this amount is considerably small compared to the total volume of the melt and is thus neglected.

During the Limited Growth period the bubble growth rate quickly reaches a maximum and decreases. The increased diffusion leads to a decrease in concentration gradient resulting in a decrease in bubble growth rate. This starts the slowing of the bubble growth until the cellular structure is frozen when $T_{g}>T_{a m b}$. 
It is interesting to note that the bubble still has considerable pressure when the growth completely stops. This allows the possibility to add a second stage to the foaming process where the temperature can be increased beyond the $T_{g}$ of the plasticized polymer and cause further foaming (Costeux and Zhu, 2013).
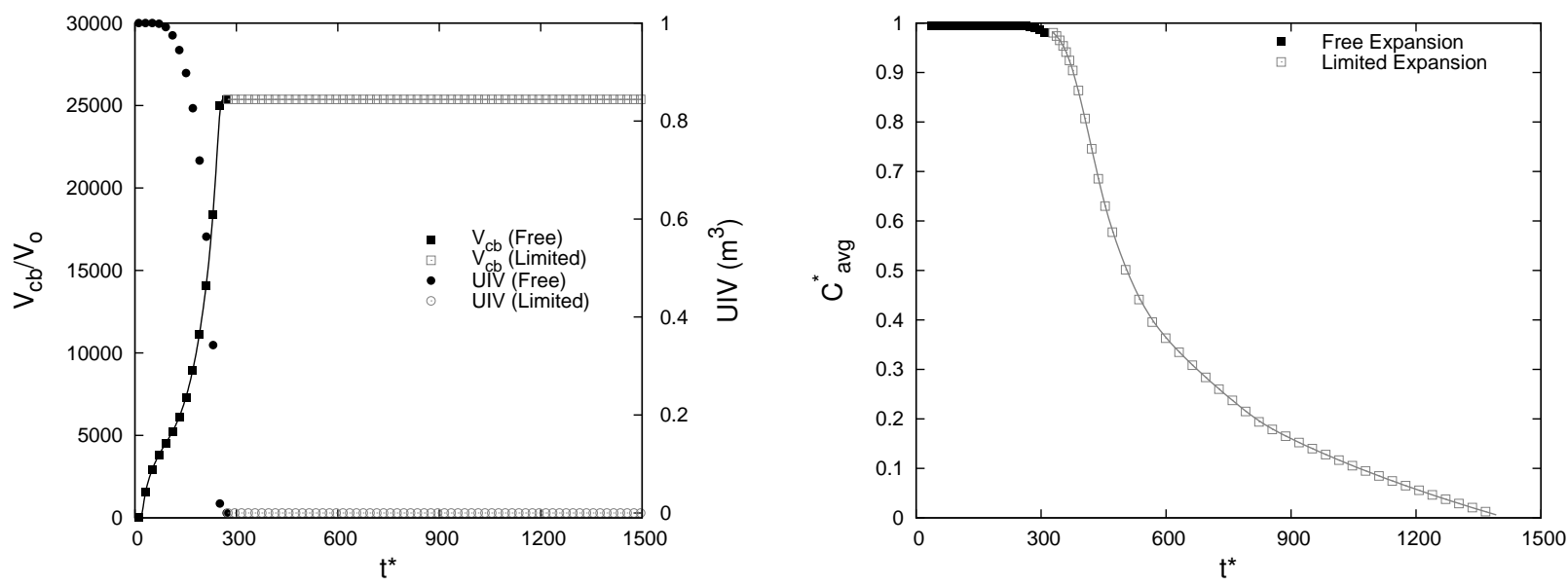

(a) Variation of bubble Influence Volume and total Un-(b) Average blowing agent concentration $\left(C_{\text {avg }}^{*}\right)$ w.r.t influence Volume w.r.t time time

Figure 6: Variation of the bubble parameters w.r.t time.

\section{Comparison to experimental data}

The rationale behind the choice of the polymer and additional details on the experimental setup and methodology for obtaining the cell size distribution from the nanocellular foam samples can be found in Costeux et al. (2014). For completeness, the batch foaming process of the MMA-EA9 polymer is briefly described here. The $3 \mathrm{~mm}$ thick samples are soaked with supercritical 
$\mathrm{CO}_{2}$ at selected temperature $\left(T_{\text {soak }}\right)$ and pressure $\left(P_{\text {soak }}\right)$. The soaking takes place inside a pressurized vessel for 6 hours to ensure complete saturation of the polymer with $\mathrm{CO}_{2}$. Subsequently, the vessel is rapidly depressurized to initiate nucleation and growth of $\mathrm{CO}_{2}$ bubbles inside the polymer. The growth in cell size is arrested when the glass transition temperature of the polymer $+\mathrm{CO}_{2}$ system rises above the ambient temperature.

The depressurization experiments were carried out for sample soaking conditions of $30 \mathrm{MPa}-35^{\circ} \mathrm{C}, 30 \mathrm{MPa}-55^{\circ} \mathrm{C}, 36 \mathrm{MPa}-35^{\circ} \mathrm{C}, 36 \mathrm{MPa}-55^{\circ} \mathrm{C}$ and $33 \mathrm{MPa}-$ $45^{\circ} \mathrm{C}$. For each of the soaking conditions two foam samples were made. One sample was immersed immediately after depressurization in an iced water bath at $0^{\circ} \mathrm{C}$ to arrest cell growth. The other sample was left at ambient temperature $25^{\circ} \mathrm{C}$ to allow foam stabilization. It was found that the samples left at $25^{\circ} \mathrm{C}$ had a slightly lower density and larger mean cell size, indicating a phenomenon of slow cell growth with minimal coalescence.

In order for the foaming model to match the cell density and cell size distribution, the model should use the right depressurization profile which in turn captures the correct nucleation rate and total nucleation time. Figure 7 shows the measured depressurization profile from the batch foaming experiments for the samples soaked at 30MPa. It also shows an exponential depressurization profile that is a reasonable match with the measured profile. In the following work on comparison with experimental data (section 5, the exponential profile is employed to reduce the additional computational cost of interpolating the pressure data for every timestep from the measured values. The figure also shows a linear depressurization rate more representative of a continuous extrusion process. 


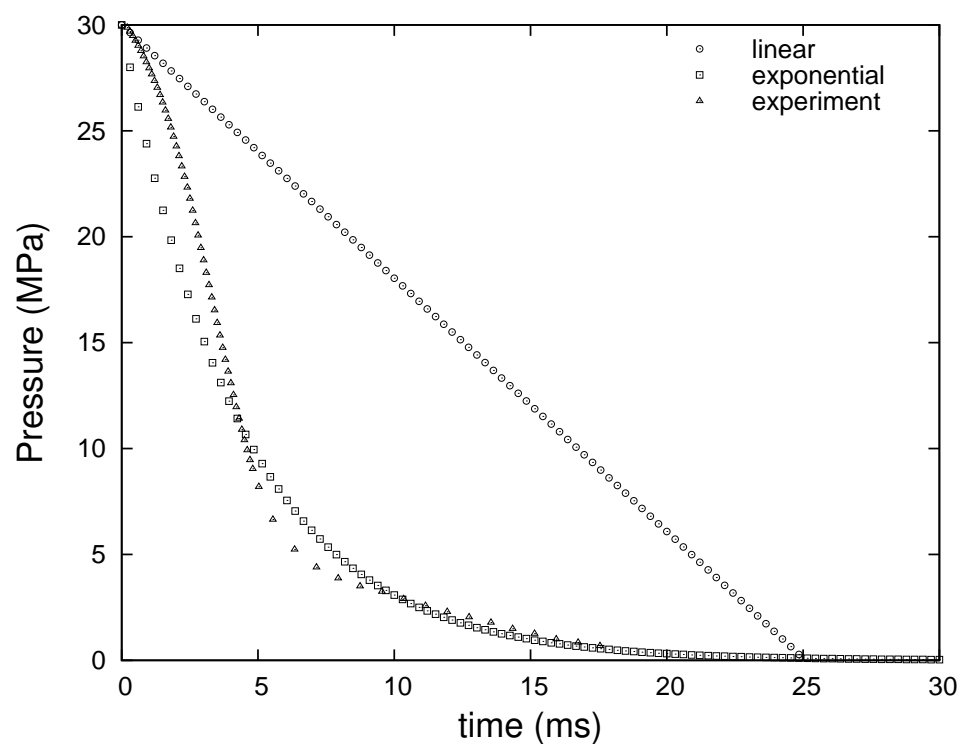

Figure 7: Comparison of the linear and exponential depressurization rate profiles to the measured profile from experiments. 


\subsection{Setting CNT model parameters from experimental data}

As mentioned earlier, Equation 11 which governs nucleation rate, contains two parameters $\left(A_{1}\right.$ and $\left.A_{2}\right)$, which have to be determined from experimental data. The nucleation rate is proportional to $A_{1}$, which can be considered as a representation of density of available nucleation sites. Increase the value of $A_{1}$ results in an increase in the nucleation rate.

Nucleation requires overcoming an energy barrier that is affected by the parameter $A_{2}$. Increasing $A_{2}$ delays the onset of nucleation. The effect of such a delay on the BSD depends on the depressurization profile. Figure 8 shows the plot of nucleation rate with respect to time as $A_{2}$ varies, for a model polymer $+\mathrm{CO}_{2}$ system. The depressurization rate is assumed to be constant in this plot. As the value of the parameter $A_{2}$ increases not only is the onset of nucleation delayed but the rate of increase in nucleation also slows down. If a constant depressurization rate is assumed, such an increase in $A_{2}$ results in increasing the breath of the BSD, thus creating larger bubbles.

The experimental data available for the determination of $A_{1}$ and $A_{2}$ consist of cell size and porosity (foam void volume fraction). Experimental data capturing the onset and end of nucleation would be very helpful for the direct calculation of $A_{2}$, but cannot practically be measured for such rapid nanocellular foaming process. Thus $A_{1}$ and $A_{2}$ were obtained by matching the cell size and porosity data of foams made at $30 \mathrm{MPa}-35^{\circ} \mathrm{C}$ and $30 \mathrm{MPa}-55^{\circ} \mathrm{C}$. Note that this is the minimum amount of data that can be used to estimate $A_{1}$ and $A_{2}$. This resulted in a value for $A_{1}=4.5 \times 10^{-24}$ and $A_{2}=67.2$. The same values of $A_{1}$ and $A_{2}$ were used for all other model predictions at other temperatures and pressures. 


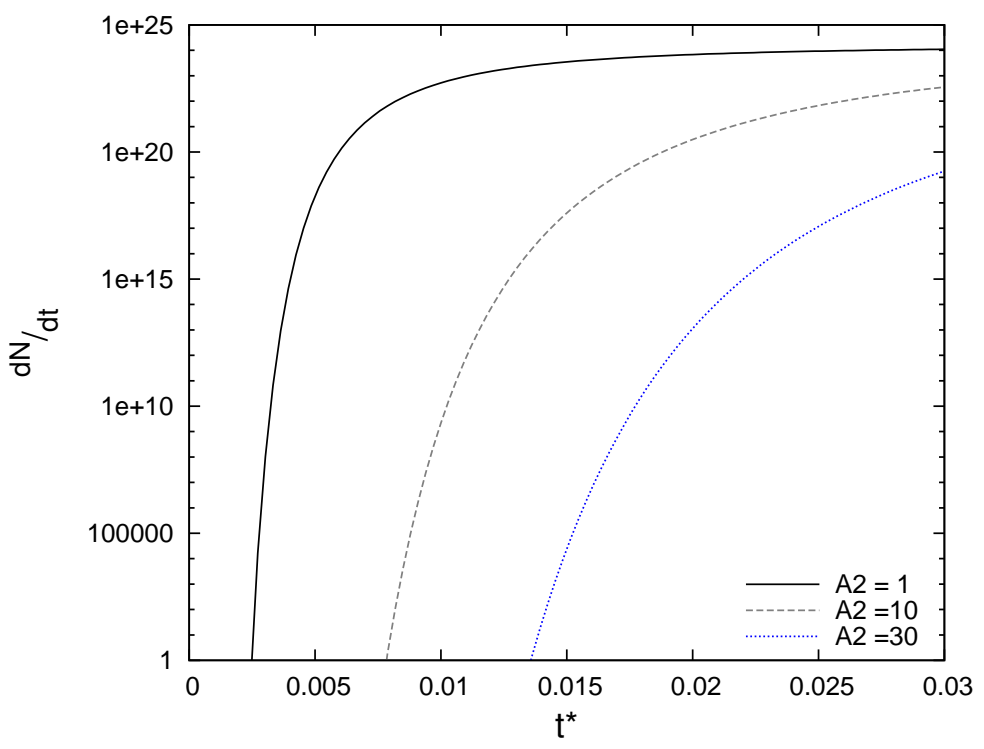

Figure 8: Increasing the parameter $A_{2}$ in CNT model causes a delay in the onset of nucleation as well as a lower nucleation rate. 


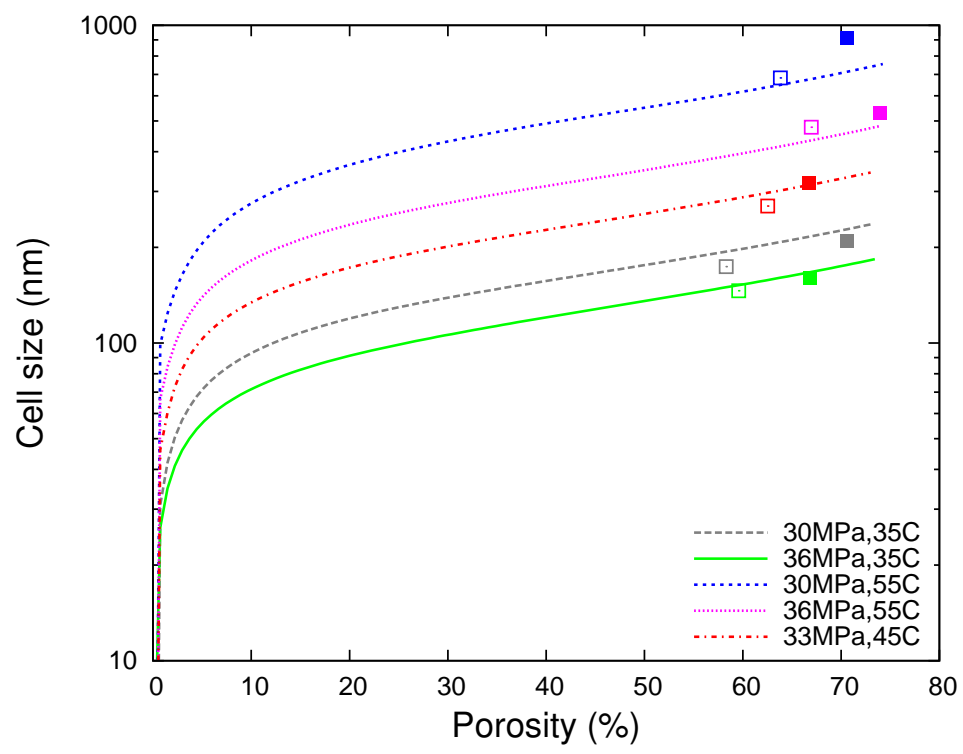

Figure 9: Comparison of the foaming model results with experimental data for MMA-EA9. Experimental data is provided as closed symbols $\left(25^{\circ} \mathrm{C}\right)$ and open symbols $\left(0^{\circ} \mathrm{C}\right)$. 
Figure 9 plots the model predictions of average cell size with respect to porosity during the foaming of MMA-EA9 with $\mathrm{CO}_{2}$ as the blowing agent at different soak conditions. The plot also shows experimental data for samples foamed at the same soaking conditions. Open symbols represent sample immersed in water at $0^{\circ} \mathrm{C}$ and closed symbols represent samples left at ambient conditions $\left(25^{\circ} \mathrm{C}\right)$, immediately after depressurization.

The model captures the effect of soak temperature and soak pressure in predicting the average cell size accurately. The close model predictions indicate that the model is accurately predicting the total bubbles nucleated at different conditions. The final porosity and cell size predicted by the model (end of the lines in Figure 9) exceed the experimental values. This indicates that the criterion used to arrest foam expansion, namely $T_{g}$ exceeding the ambient temperature, allows for more expansion than observed experimentally. This is clearly evident when the cell size of foams quenched at $0^{\circ} \mathrm{C}$ after depressurization are compared to the model prediction. A likely explanation is the internal cooling that takes place in the foam due to rapid $\mathrm{CO}_{2}$ expansion. It should be noted that the cooling does not seem to impact the total number of nuclei generated, because the isothermal model correctly predicts the foam expansion pathway. This can be explained by the fact that nucleation time is short (fraction of the depressurization time), and does not allow the sample to cool. The main impact is on the final expansion, as foam stabilization occurs earlier than expected from the isothermal model. Samples not quenched at $0^{\circ} \mathrm{C}$ but maintained at $25^{\circ} \mathrm{C}$ after depressurization retain the ability to expand further, along the same growth curve, as seen by the increase in cell size and porosity of those foams in Figure 9. Further 
iterations of the model will attempt to account for internal cooling and its effect on cell growth and foam expansion.

Figure 10 shows the comparison of the bubble size distribution for samples soaked at $30 \mathrm{MPa}-35^{\circ} \mathrm{C}$ and $30 \mathrm{MPa}-55^{\circ} \mathrm{C}$ with the model data. The bubble size distribution is initially reprepresented with a histogram of number fractions $N_{i}^{*}$ and bin boundaries $b_{i}$, such that $N_{i}^{*}$ is the number fraction of bubbles with a radius of $b_{i} \leq R_{i}<b_{i+1}$. Since the model data has many more bins thatn the experimental data, the histogram data are more easily compared on the same scale after conversion to a discrete number density function $N_{D F}^{i}$, defined as,

$$
N_{D F}^{i}=\frac{N_{i}^{*}}{b_{i+1}-b_{i}}
$$

This choice is normalized, since

$$
\sum_{i=1}^{\infty} N_{D F}^{i}\left(b_{i+1}-b_{i}\right)=1
$$

The BSD predicted by the model is in good agreement with the experimental BSD for both foams generated at a pressure of $30 \mathrm{MPa}$. In the case of $30 \mathrm{MPa}-35^{\circ} \mathrm{C}$, the model over-predicts the average cell size, as also seen in Figure 9, yet the predicted BSD profile is very similar to experimental data, with a distribution slightly skewed towards smaller cell sizes. For the $30 \mathrm{MPa}-55^{\circ} \mathrm{C}$ case, the distribution is less skewed, and the model matches the experimental data very well.

Figure 11 shows the comparison of the predicted and experimental BSD for the $36 \mathrm{MPa}$ case at $35^{\circ} \mathrm{C}$ and $55^{\circ} \mathrm{C}$.. The agreements is good at both 


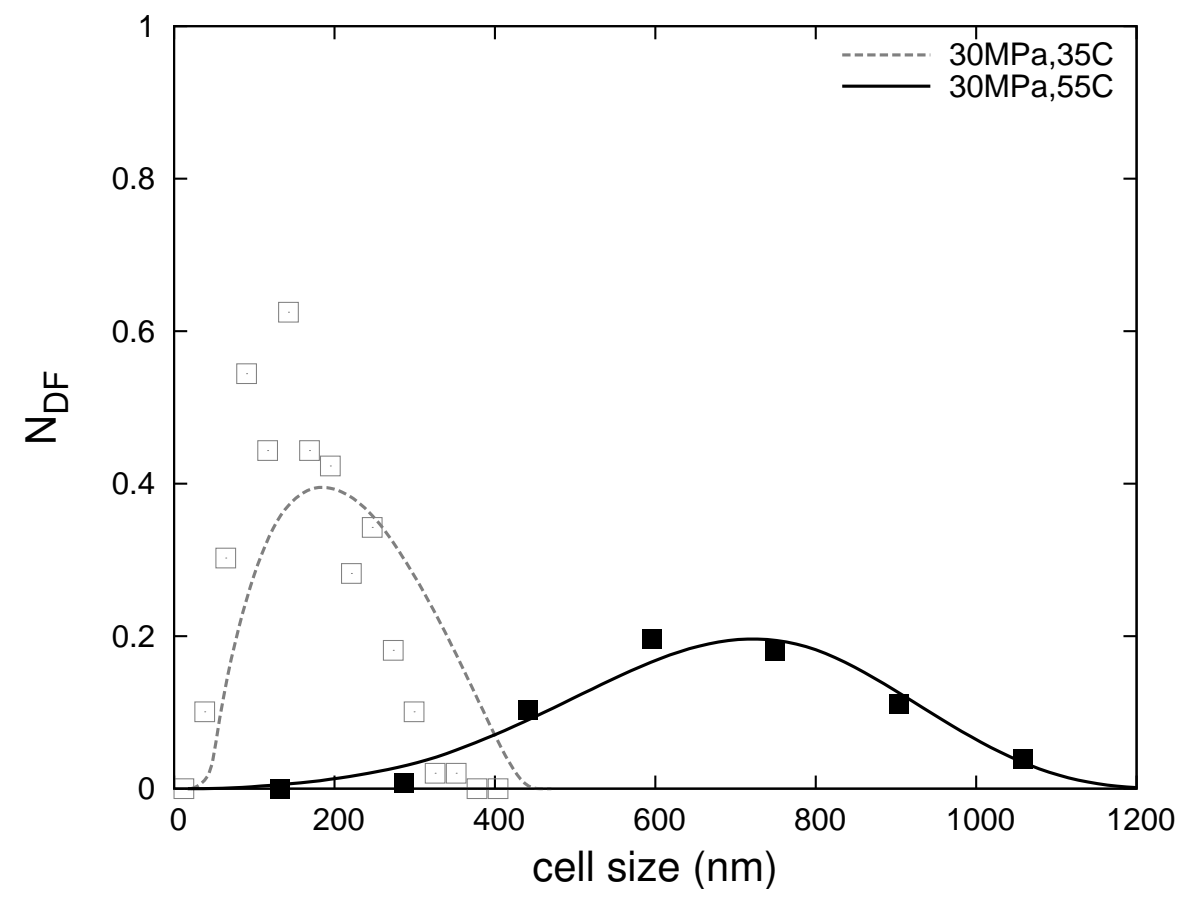

Figure 10: Comparison of BSD predicted by the model to the experimental data. Samples were soaked at $30 \mathrm{MPa}, 35^{\circ} \mathrm{C}(\square)$ and $30 \mathrm{MPa}, 55^{\circ} \mathrm{C}(\boldsymbol{\square})$. 


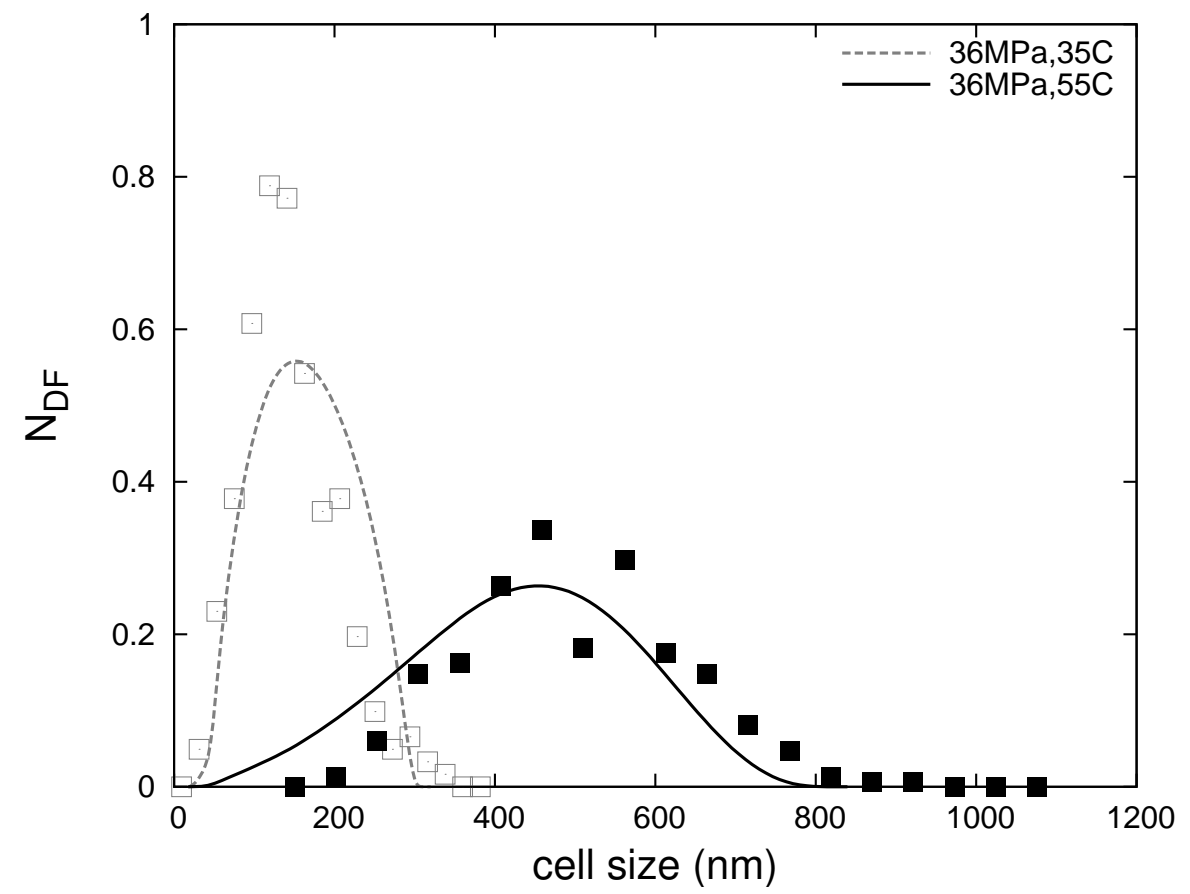

Figure 11: Comparison of BSD predicted by the model to the experimental data. Samples were soaked at $36 \mathrm{MPa}, 35^{\circ} \mathrm{C}(\square)$ and $36 \mathrm{MPa}, 55^{\circ} \mathrm{C}(\mathbf{\square})$.

temperature, although the model also slightly over-predicts the average cell size in the $35^{\circ} \mathrm{C}$ case, as it did at the lower pressure.

Both plots show that the model can predict the effect of both temperature and pressure on the mean bubble size and the BSD profile with with good accuracy.

The general behavior of the BSD can be understood from Figure 12 which shows the depressurization profile over time and the parameter $\left(\zeta / \zeta_{\max }\right)$, where $\zeta$ is given as

$$
\zeta=\frac{d N}{d t} U I V \Delta t
$$




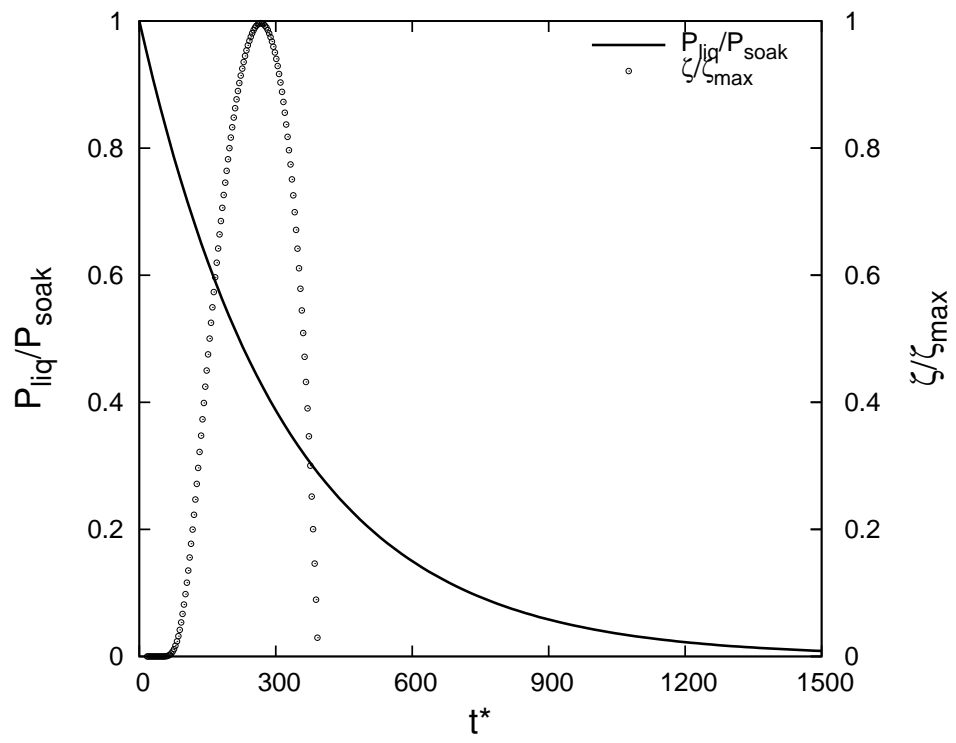

Figure 12: Growth of bubbles nucleated at different stages of nucleation.

and $\zeta_{\max }$ is the maximum value that $\zeta$ attains. The data for this plot was generated for the foaming of $30 \mathrm{MPa}-35^{\circ} \mathrm{C}$ case. When nucleation starts, $\frac{d N}{d t}$ is small due to a small value of $P_{\text {sat }}-P_{\text {liq }}$ which drive the nucleation rate. This leads to fewer bubbles being generated in this period but these bubbles grow the most and eventually reach the largest cell size. Consequently, this initial period produces the high end of the BSD. During the nucleation period $P_{\text {sat }}$ does not change significantly, since the amount of dissolved blowing agent in the polymer remains almost constant. Thus the value of $P_{s a t}-P_{l i q}$ increases over the nucleation time, which leads to an increase in $\frac{d N}{d t}$ and $\zeta$ that produces the peak in the BSD. Towards the end of nucleation $P_{\text {sat }}-P_{l i q}$ remains high but the available UIV keeps decreasing towards zero, which results in a rapid decrease of the nucleation rate. This latest stage produces the BSD tail at the lower bubble size end. 


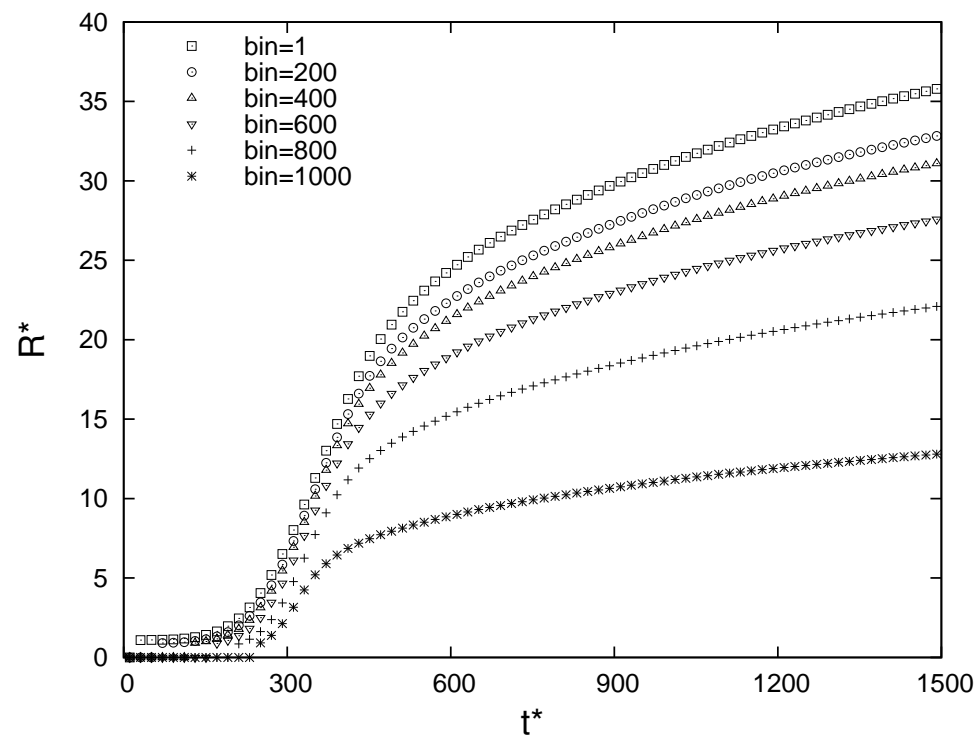

Figure 13: Growth of bubbles nucleated at different stages of nucleation.

However, it is important to note that all the nuclei do not grow at the same rate. The depressurization profile, the amount of blowing agent left in the melt phase and the timeframe of bubble nucleation determine its growth rate. Figure 13 shows the growth of bubbles in various bins. Each bin $b_{i}$ represents the bubble nucleated in between time $t_{i}$ and $t_{i+1}$. The figure shows that bubbles nucleated during the beginning of nucleation tend to grow faster and larger as opposed to bubble that nucleated later. Bubbles nucleated in the beginning experience faster depressurization and a polymer that is more plasticized by $\mathrm{CO}_{2}$ than the bubbles that nucleate later.

Note that for the case of $30 \mathrm{MPa}-35^{\circ} \mathrm{C}$ and to some extent $36 \mathrm{MPa}-35^{\circ} \mathrm{C}$, the experimental data shows that the BSD is similar to a log-normal distribution. The foaming model also shows the same skewed distribution represented by a log-normal distribution. Two factors help explain the log-normal distri- 
bution. Firstly as Figure 13 shows bubbles nucleation at different times have different growth rates, secondly, there is a rapid drop in nucleation rate at the end of nucleation compared to the gradual increase in nucleation during the start of nucleation as shown in Figure 12.

Despite a number of approximations, the foaming model based on the IVA and CNT successfully predicts the BSD because it captures the start and end of nucleation along with the changing nucleation rates during the foaming process accurately.

\section{Effect of depressurization curve on bubble size distribution}

The above results point to the importance of the depressurization curve on the BSD profile. However the actual depressurization profile that the polymer and blowing agent system experiences depends on the process and equipment chosen. In the following the BSD obtained from different depressurization profiles are compared.

Three depressurization profiles with the same depressurization time, linear, exponential and experimentally measured previously shown in Figure 7, were used for the foaming simulations at $30 \mathrm{MPa}-35^{\circ} \mathrm{C}$. The linear profile could possibly represent a continuous extrusion process. Although the exponential and the measured depressurization profile represent the same batch foaming process, there are some differences between the two profiles which warranted the comparison. The parameters $A_{1}$ and $A_{2}$ were kept the same, as mentioned earlier.

Figure 14 shows the normalized BSD given by the parameter $N_{D F}$ for the three depressurization profiles. It is seen that the linear profile leads to 


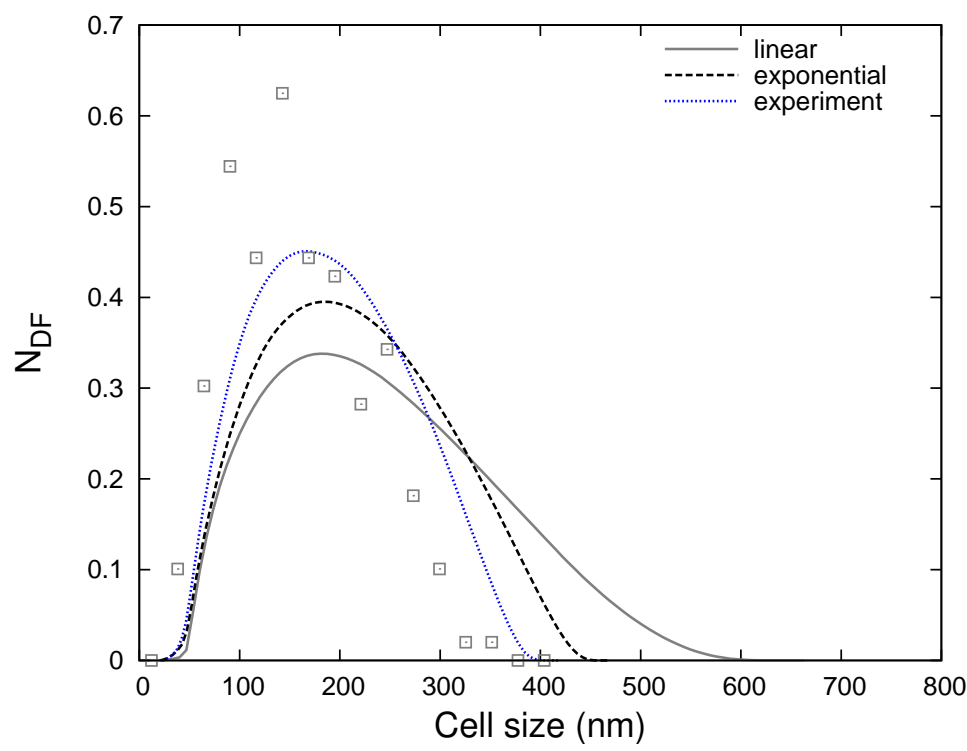

Figure 14: Effect of depressurization profile on cell size distribution; experimental data is represented by $\bullet$.

broader BSD with the mean bubble size of $263 \mathrm{~nm}$. The exponential profile has a slightly narrower distribution with a mean bubble size of $237 \mathrm{~nm}$. The measured profile shows an even narrow distribution with mean bubble size of $215 \mathrm{~nm}$ and also provides the best match with experimental data.

In order the understand the behavior of the BSD shown in Figure 14, it is important to know that given a fixed polymer and blowing agent system, lower nucleation rates lead to fewer nuclei overall, a direct consequence of the IVA. Thus it is clear that the linear profile leads to the lowest total number of nuclei and thus the largest mean bubble size due to the nucleation rate being dependent on depressurization rate. The difference in depressurization rates between the exponential and experimentally measured profiles is less significant . However, Figure 12 shows that nucleation rate is the highest when 
$P / P_{\text {soak }}$ is between 0.7 and 0.2 . In this range of pressure, the depressurization rate of the experimental profile is higher than that of the exponential profile. As a result, the predicted density of nuclei is higher and the final average cell size smaller when depressurization follows the experimentally measured profile.

\section{Conclusions}

The goal of this work was to address the lack of a comprehensive foaming model capable of providing BSD for nanocellular foams produced by batch foaming as a function of processing conditions and material properties. This is true particularly for processing at very high depressurization rate.

A model was created, which incorporates a semi-empirical nucleation equation derived from the CNT, coupled with bubble growth equations using the influence volume approach. The analysis of a single bubble growth during such high depressurization reveals the presence of two distinctly different growth stages. The first stage is a rapid cell growth period, which occurs at the beginning of the cell growth process and is mainly driven by pressure difference between the bubble interior and instantaneous external pressure. The growth in this stage is insensitive to the $\mathrm{CO}_{2}$ diffusivity, but seems to depend more strongly on the viscosity or elasticity of polymer melt, and hence this period can be referred to viscosity-controlled.

The decrease in the bubble growth rate coincides with the transition from Free expansion period to Limited expansion period. The slow growth during Limited Expansion period is driven by diffusion of blowing agent from the polymer + gas mixture into the bubble. Unlike in micro-cellular foams it 
is found that the diffusion controlled period is relatively short, being only a few times longer than the time-scales for viscosity controlled period. This is due to the rapid stabilization of nano-cellular foams caused by a foaming temperature below to the $T_{g}$ of the polymer, whereas microcellular foams are usually produced above $T_{g}$.

Despite the simplifying assumptions of the foaming model and approximations in acquiring data for physical properties, the model is found to predict the cell density and cell size distribution with excellent accuracy. However, this has been possible only after obtaining two CNT parameters by adjusting the average cell size result to experiments at one pressure and two temperatures. It is found that the two parameters $A_{1}$ and $A_{2}$ together control the onset and end of nucleation during depressurization, and also the nucleation rate. Once these parameters have been determined for a given system of polymer and blowing agent, the model predicts average cell size at other temperature and pressure conditions, but more importantly provides an excellent approximation of the BSD, in particular if the depressurization profile matches that used in the experiments.

The new insights into the foaming mechanism at the nanoscale were used to explain the effect of the shape of the depressurization profile on the BSD. It was found that for the same total depressurization time using a linear depressurization profile, more typical of extrusion foaming processes, leads to a broader cell size than for an exponential depressurization profile due to the lower peak depressurization rate. Such information can be used to improve the design of existing process and equipment to design nano-cellular foams with optimal BSD. 


\section{Acknowledgments}

We thank The Dow Chemical Company for permission to publish this work, Dr. Shana Bunker (Dow Building \& Construction) for providing part of the experimental data, and Argonne National Laboratories for access to their computing resources (Fusion) to carry out the computationally expensive simulations.

\section{Funding}

This material is based upon work that was in part supported by the U. S. Department of Energy under Award Number DE-EE0003916.

\section{Disclaimer}

This report was prepared as an account of work sponsored by an agency of the United States Government. Neither the United States Government nor any agency thereof, nor any of their employees, makes any warranty, express or implied, or assumes any legal liability or responsibility for the accuracy, completeness, or usefulness of any information, apparatus, product, or process disclosed, or represents that its use would not infringe privately owned rights. Reference herein to any specific commercial product, process, or service by trade name, trademark, manufacturer, or otherwise does not necessarily constitute or imply its endorsement, recommendation, or favoring by the United States Government or any agency thereof. The views and opinions of authors expressed herein do not necessarily state or reflect those of the United States Government or any agency thereof. 


\section{References}

Amon, M., Denson, C., 1984. A study of the dynamics of foam growth: Analysis of the growth in closely spaced spherical bubbles. Polymer Engineering and Science 24 (13), 1026-1034.

Arefmanesh, A., Advani, S., Michaelides, E., 1992. An accurate numerical solution for mass diffusion-induced bubble growth in viscous liquids containing limited dissolved gas. International Journal of Heat and Mass Transfer. $35,1711-1722$.

Blander, M., Katz, J., 1975. Bubble nucleation in liquids. AIChE Journal $21(5), 833-848$.

Condo, P., Johnston, K., 1994. In site measurement of the glass transition temperature of polymers with compressed fluid diluents. Journal of Polymer Science, Part B: Polymer Physics 32, 523-533.

Costeux, S., 2014. $\mathrm{CO}_{2}$-Blown Nanocellular Foams. Journal of Applied Polymer Science 131 (23), 41293.

Costeux, S., Bunker, S., Jeon, H., 2013. Homogeneous nanocellular foams from styrenic-acrylic polymer blends. Journal of Materials Research 28, $2351-2365$.

Costeux, S., Khan, I., Jeon, H., Bunker, S., 2014. Experimental study and modeling of nanofoam formations from single phase acrylic copolymer systems. Journal of Cellular Plastics doi:10.1177/0021955X14531972. 
Costeux, S., Zhu, L., 2013. Low density thermoplastic nanofoams nucleated by nanoparticles. Polymer 54, 2785-2795.

Feng, J., Bartelo, C., 2004. Prediction of bubble growth and size distribution in polymer foaming based on new heterogeneous nucleation model. The Society of Rheology, Inc. 48 (2), 439-462.

Forest, C., Chaumont, P., Cassagnau, P., Swoboda, B., Sonntag, P., 2014. Polymer nano-foams for insulating applications prepared from $\mathrm{cO}_{2}$ foaming. Progress in Polymer Science 41, 122.

Han, C., Yoo, H., 1981. Studies on structural foam processing 4. Bubblegrowth during mold filling. Polymer Engineering and Science 21, 518-533.

Han, J., Han, C., 1990. Bubble nucleation in polymeric liquids. 1. Bubble nucleation in concentrated polymer solutions. Journal of Polymer Science. $28,711-741$.

Kim, Y., Park, C., Chen, P., Thompson, R., 2011. Origins of the failure of classinal nucleation theory for nanocellular polymer foams. Soft Matter 7, $7351-7358$.

Lee, J., 1995. Controlled structure processing of cellular materials. Ph.D. thesis, Texas A\&M University.

Leung, S., Li, H., Park, C., 2007. Impact of approximating the initial bubble pressure on cell nucleation in polymeric foaming processes. Journal of Applied Polymer Science 104 (902-908). 
Leung, S., Park, C., Li, H., 2006a. Numerical simulation of polymeric foaming processes using modified nucleation theory. Plastics, Rubber and Composites $35,93-100$.

Leung, S., Park, C., Xu, D., Li, H., Fenton, R., 2006b. Computer simulation of bubble-growth phenomena in foaming. Ind. Eng. Chem. Res. 45, 78237831.

Mao, D., Edwards, J., Harvey, A., 2006. Prediction of foam growth and its nucleation in free and limited expansion. Chemical Engineering Science 61, 1836-1845.

Nilsson, F., Hallstensson, K., Johansson, K., Umar, Z., Hedenqvist, M. S., 2013. Predicting solubility and diffusivity of gases in polymers under high pressure: N2 in polycarbonate and poly(ether-ether-ketone). Industrial and Chemical Engineering Research 52 (26), 8655-8663.

Notario, B., Pinto, J., Solorzano, E., de Saja, J., Dumon, M., RodriguezPerez, M., 2014. Experimental validation of knudsen effect in nanocellular polymeric foams. Polymer 56, 57-67.

Park, C., Baldwin, D., Suh, N., 1995. Effect of pressure drip rate on cell nucleation in continuous processing of microcellular polymers. Polymer Engineering and Science. 35, 432-440.

Park, H., Dealy, J., 2006. Effects of pressure and supercritical fluids on the viscosity of polyethylene. Macromolecules 39, 5438-5452. 
Payvar, P., 1987. Mass transfer-controlled bubble growth during rapid decompression of a liquid. International Journal of Heat and Mass Transfer 30, 699-706.

Shafi, M., Joshi, K., Flumerfelt, R., 1997. Bubble size distributions in freely expanded polymer foams. Chemical Engineering Science 52, 635-644.

Shafi, M., Lee, J., Flumerfelt, R., 1996. Prediction of cellular structure in free expansion polymer foam processing. Polymer Engineering and Science 36 (14), 1950-1959.

Shimoda, T., Tsujimura, I., Tanigaki, M., Ohshima, M., 2001. Polymeric foaming simulation for extrusion processes. Journal of Cellular Plastics 37, $517-536$.

Street, J., 1968. The rheology of phase growth in elastic liquids. Transactions of the Society of Rheology 12, 103-131.

Street, J., Fricke, A., Reiss, L., 1971. Dynamics of phase growth in viscous, non-newtonian liquids. Industrial \& Engineering Chemistry Fundamentals $10,54-64$.

Taki, K., 2008. Experimental and numerical studies on the effects of pressure release rate on number density of bubbles and bubble growth in a polymeric foaming process. Chemical Engineering Science 63, 3643-3653.

Taki, K., Yatsuzuka, T., Ohshima, M., 2003. Visual observations of batch and continuous foaming process. Journal of Cellular Plastics. 39, 155. 
Venerus, D., Yala, N., Bernstein, B., 1998. Analysis of diffusion-induced bubble growth in viscoelastic liquids. Journal of Non-Newtonian Fluid Mechanics. 75, 55-75.

Xu, X., Cristancho, D., Costeux, S., Wang, Z., 2012. Density-functional theory for polymer-carbon dioxide mixtures: A perturbed-chain saft approach. The Journal of Chemical Physics 137 (054902).

Xu, X., Cristancho, D., Costeux, S., Wang, Z., 2013a. Bubble nucleation in a polymer- $\mathrm{CO}_{2}$ mixtures. Soft Matter 9 (40), 9675-9683.

Xu, X., Cristancho, D., Costeux, S., Wang, Z., 2013b. Discontinuous bubble nucleation due to a metastable condensation transition in a polymer- $\mathrm{CO}_{2}$ mixtures. Journal of Physical Chemistry Letters 4 (10), 1639-1643. 